\title{
Activity-Feedback Adaptive Particle Swarm Optimization
}

\author{
Genki Ueno and Keiichiro Yasuda \\ Dept. of Electrical Engineering, Tokyo Metropolitan University \\ 1-1, Minamiosawa, Hachioji-shi, Tokyo 192-0397, Japan \\ E-mail: yasuda@eei.metro-u.ac.jp
}

\begin{abstract}
This paper proposes an adaptive Particle Swarm Optimization (PSO) algorithm using the information defined as the average absolute value of velocity of all of the particles, which information can be used as an index to understand the activity of all of the particles. While a stability analysis of PSO algorithm is carried out based on the stability theory of modern control theory, an adaptive strategy for tuning one of its parameters is introduced so as to follow a given ideal average velocity by feedback control. The feasibility and advantages of the proposed adaptive PSO algorithm are verified through numerical simulations using some typical global optimization problems.
\end{abstract}

\section{Introduction}

The Particle Swarm Optimization (PSO) algorithm, which is one of the most powerful algorithms available for solving unconstrained global optimization problems, was originally proposed by J. Kennedy et al. as an optimization method in 1995. Subsequent numerical experiments have demonstrated that the PSO algorithm is efficient for a variety of global optimization problems with nonlinear and multi-peaked features [1].

Metaheuristics, such as Genetic Algorithms, Simulated Annealing, Tabu Search, PSO and so on, are mainly designed to be applied to engineering problems. These algorithms should be applicable to several types of real optimization problems without the need for highly skilled labor. However, to date, their application has required significant time and labor for the tuning of parameters. Therefore, from an engineering viewpoint, it is desirable to add adaptability to these algorithms. The adaptability of an optimization algorithm can be defined as an ability to guarantee appropriate adaptable adjustment of the algorithm's parameters with respect to predetermined structural variation of problems to be solved.

The adaptability property is especially important from the viewpoint of practical application of Metaheuristics. In order to add adaptability to an optimization algorithm, two significant relationships must be understood. First, the qualitative and quantitative relationship between the parameters and the behavior of the algorithm must be analyzed. Second, the qualitative and quantitative relationship between the behavior of the algorithm and the success, or failure, of the search must be analyzed.

The modification of the algorithm due to the results of these analyses should be carefully weighted so that an ideal algorithm behavior may be determined relative to the success of the search, and so that an adaptive algorithm which feeds back the conditions of the search in order to maintain this ideal behavior may be constructed.

However, analytical research to examine the dynamics of PSO in the search process remains insufficient, and relatively little is known about how the PSO algorithm works or finds a globally optimal solution to a problem. The dynamics of PSO in the search process was investigated by M. Clerc and J. Kennedy based on a simplified model of PSO. They proposed and analyzed a model that uses three types of simplification: (1) randomness is removed, (2) gbest, the best position of the swarm, and pbest, the best position of each particle (search point), are combined into one term comprising their weighted average, and (3) this dynamic weighted average of the two best positions is modeled to be static.

Although a significant function of the multipoint search, i.e. the interaction among particles of PSO, cannot be taken into consideration in the above simplification, this important research represents the first analysis of the dynamics of PSO.

Based on the previous study [2], in the present study, we commence our analysis of the dynamics of PSO using the same simplified model. In considering the qualitative and quantitative relationship between the behavior of the algorithm and the success or failure of the search, we examine the qualitative relationship between the average velocity of particles of PSO and the success or failure of the search. This is achieved by considering the results of numerical simulations for various benchmark problems. Finally, we propose an adaptive PSO that feeds back the information of the average velocity of particles, and we verify the search ability of the proposed algorithm through numerical simulations using some typical global optimization problems.

\section{Particle Swarm Optimization}

\subsection{Desirable features of metaheuristics}

The PSO algorithm is one of the optimization algorithms included among the algorithms of metaheuris- 


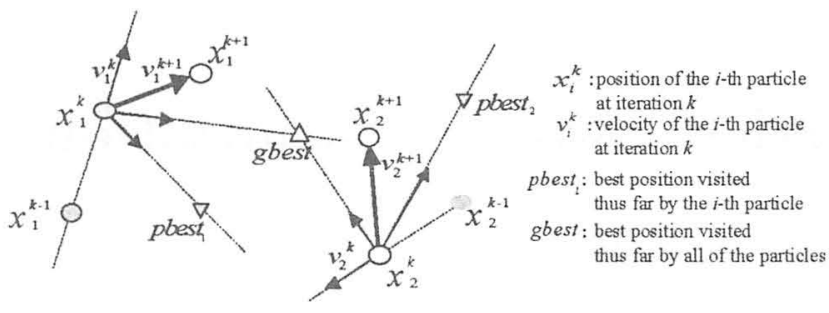

Fig. 1: Particle movement

tics, a group of approximate algorithms that aim to obtain approximate solutions within a practical calculation time. Generally, metaheuristics contain parameters. If appropriate parameters are chosen for the search, then good results can be obtained; otherwise, the search often ends in failure. Metaheuristics should have the following three capabilities:

(1) Adjustability: an ability to guarantee the qualitative and quantitative relationships between parameters in an algorithm and the behavior of the algorithm.

(2) Robustness: an ability to guarantee the performance of a search for pre-adjusted parameters with respect to the predetermined structural variation of problems to be solved.

(3) Adaptability: an ability to guarantee appropriate adaptable adjustment of the algorithm's parameters with respect to predetermined structural variation of problems to be solved.

An algorithm possessing the adjustability described in (1) may be able to be transformed to an algorithm possessing the adaptability described in (3). The analysis of the present study, described below, improves the understanding of the qualitative relationship between parameters and the dynamics of particles.

Based on this analysis, a new algorithm that takes adaptability into account will be proposed herein.

\subsection{Background and Outline of PSO Algorithm}

The PSO algorithm employs a swarm of multiple particles, each of which has its own position and velocity (transfer vector). The multi-particle-based search technique distinguishes the PSO algorithm from many of the other previously proposed optimization algorithms. All of the particles share information obtained from the other particles, and interaction among the particles makes the search efficient. Although only simple operations compose the PSO algorithm, the PSO algorithm can solve both nonlinear and multi-peaked optimization problems efficiently.

Although both binary-number and real-number algorithms exist, only PSO in real-number form is analyzed in the present paper. Although PSO was originally constructed as part of an investigation of the movement of a swarm in two-dimensional space, PSO as an optimization algorithm can work in optional $n$-dimensional space.

Each particle has its own position, $x$, transfer vector, $v$, and own best position visited thus far, pbest. Furthermore, all of the particles share the best position visited by all of the particles thus far, gbest. A particle forms a new transfer vector by linearly combining three vectors. In the $(k+1)$-th transfer, the $j$-th coordinate component of the transfer vector of the $i$-th particle is manipulated according to the following equation:

$$
\begin{aligned}
v_{i j}^{k+1}=w \cdot v_{i j}^{k} & +c_{1} \cdot \operatorname{rand}_{1}() \cdot\left(\text { pbest }_{i j}^{k}-x_{i j}^{k}\right) \\
& +c_{2} \cdot \operatorname{rand}_{2}() \cdot\left(\text { gbest }_{j}^{k}-x_{i j}^{k}\right)
\end{aligned}
$$

where $i=1, \cdots, m$ ( $m$ is the size of the swarm), $j=1, \cdots, n$ ( $n$ is the size of space of a given problem), $w, c_{1}$ and $c_{2}$ are positive constants, rand() is a random number that is uniformly distributed in $[0,1]$, and $k$ determines the iteration number. Customarily, the transfer vector $v_{i j}$ is also referred to as the velocity in PSO, and the present paper follows this convention. Each particle moves according to the following equation:

$$
x_{i j}^{k+1}=x_{i j}^{k}+v_{i j}^{k+1}
$$

\subsection{Stability and behavioral features of PSO}

In this section, we describe the dependence of the dynamics of PSO upon its parameters.

The parameters $w, c_{1}$ and $c_{2}$ characterize the behavior of particles, and experience shows that the success or failure of the search is heavily dependent on the values of these parameters. The main causes of search failures are as follows:

(1) The velocity of particles increases rapidly, and particles move out of the search space,

(2) The velocity of particles decreases rapidly, and particles become immobile,

(3) Particles cannot escape from locally optimal solutions.

In order to avoid these undesirable situations, it is important to analyze the relationship between the parameters and the behavior of particles, with particular regard to the divergence and convergence of particles.

The key features of the model used in this section are described here in greater detail. The differences between the current model of PSO and actual PSO are as follows:

(a) pbest and gbest are combined to form one term, $p$

(b) $p$ is regarded as a constant,

(c) randomness is removed.

(a) is a mere algebraic transformation of pbest and gbest and does not seem to be a significant difference in and of itself.

Regarding $p$ as a constant, (b), is a significant difference between the simplified model and actual PSO. Usually, pbest and gbest are renewed in the search process. Therefore, the simplified model, in which $p$ is 


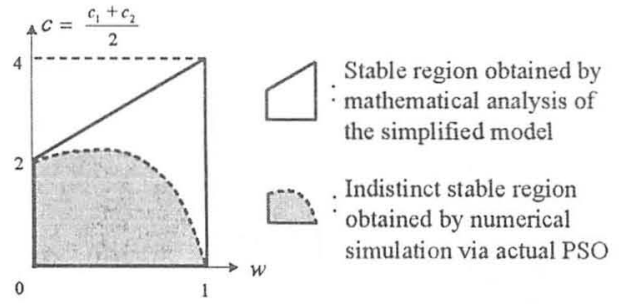

Fig. 2: Stable and unstable regions in parameter space

regarded as a constant, does not allow us to analyze the complete dynamics of PSO.

The present analysis using the simplified model indicates only that if the convergent condition is satisfied, particles will converge toward $p$ because the renewal of pbest and gbest will stop at some future time. Although we have also analyzed the stability[2], including the fluctuation of $p$, based on Bounded Input-Bounded State (BIBS) stability, the analysis is not included in the present study.

The effect of removing randomness, (c), appears to be considerable. Although a mathematical analysis including randomness has not yet been carried out, the border of convergent and divergent parameters can be roughly explored by numerical simulations. The stable and unstable regions of parameters obtained based on the conclusions of this section are shown in Figure.

In this figure, the horizontal axis indicates parameter $w$ and the vertical axis indicates the average of $c_{1}$ and $c_{2}$ $\left(c=\frac{c_{1}+c_{2}}{2}\right)$. The trapezoid indicated by the solid line is the stable region obtained by analysis of the simplified model, and the curve indicated by the broken line is the stable region obtained by simulation via actual PSO (where $c_{1}=c_{2}$ ). In the following section, an adaptive algorithm will be proposed based on these results.

\subsection{Linearly Decreasing Inertia Weight Ap- proach (LDIWA)}

Shi and Eberhart proposed an efficient strategy for tuning the parameters of PSO in 1998 [1]. In their strategy, the value of the parameter $w$ (inertia weight) is large at the beginning of the search process, whereupon it gradually becomes smaller as the iteration advances. For example, the values $w=0.9$ (at the beginning of the search process), $w=0.4$ (at the end of the search process) and $c_{1}=c_{2}=2.0$ (constant), are recommended. This strategy is referred to herein as the Linearly Decreasing Inertia Weight Approach (LDIWA). Several numerical experiments have reported that the LDIWA is effective for various types of optimization problems. According to the definition described in Section 2, the LDIWA can be interpreted as a robust strategy.

Using the strategy of the LDIWA for the adjustment of parameter $w$, while a diversification in the search is realized in the initial stage, an intensification in the search is realized in the final stage of the search. How-
Table 1: Deterioration in search ability of the LDIWA

\begin{tabular}{c|c||c||c||c||c}
\hline \hline $\begin{array}{c}\text { Dim. } \\
(\mathrm{n})\end{array}$ & $\begin{array}{c}\text { Optimal } \\
\text { value }\end{array}$ & $\begin{array}{c}\text { Mean } \\
\text { value }\end{array}$ & $\begin{array}{c}\text { Best } \\
\text { value }\end{array}$ & $\begin{array}{c}\text { Worst } \\
\text { value }\end{array}$ & $\begin{array}{c}\text { Standard } \\
\text { deviation }\end{array}$ \\
\hline \hline 20 & -1567 & -1410 & -1538 & -1256 & 62 \\
40 & -3133 & -1650 & -2370 & -780 & 399 \\
60 & -4700 & -1610 & -2666 & -1171 & 280 \\
80 & -6267 & -1850 & -2518 & -1183 & 276 \\
100 & -7833 & -2101 & -2964 & -1276 & 366 \\
\hline \hline
\end{tabular}

ever, from our numerical experiments, the search ability of the LDIWA is reduced when the scale of the problem becomes large.

Table 1 lists the best value, the worst value, the mean value and the standard deviation of the obtained best fitness $f$ (gbest ${ }^{\mathrm{T}_{\max }}$ ) of applying the LDIWA to the benchmark $2^{n}$ minima problem as formulated in Equation (3). A total of 100 runs for each problem (20, 40, $\cdots, 100$ dimensional problems) were carried out (the size of the swarm $m=20$, the maximum number of iterations $\mathrm{T}_{\max }=1000$ ). Table 1 clarifies that as the scale of problem increases, the search ability decreases. The cause of this phenomenon will be investigated in the following section.

$$
\begin{aligned}
& \text { min. } f(x)=\sum_{i=1}^{n}\left(x_{i}^{4}-16 x_{i}^{2}+5 x_{i}\right) \\
& \text { subj. to } \quad-5 \leq x_{i} \leq 5, \quad i=1, \cdots, n
\end{aligned}
$$

\section{Average Velocity of Particles}

\subsection{Index for activity of particles}

As described above, a particle transfers by a transfer vector $v_{i j}{ }^{k+1}$ generated from Equation (1); therefore, $\left|v_{i j}{ }^{k+1}\right|$ is the transfer step-size of the $i$-th particle in the $j$-th dimension at the $(k+1)$-th iteration. Since randomness is included in this equation, the transfer vector $v_{i j}$ fluctuates. The average absolute value of velocity of all of the particles can be used as an index to understand the activity of all of the particles.

$$
v_{\text {ave }}{ }^{k+1}=\frac{1}{m \cdot n} \sum_{i=1}^{m} \sum_{j=1}^{n}\left|v_{i j}{ }^{k+1}\right|
$$

The information on the average velocity of a swarm is indicated as $v_{\text {ave }}$ and is analogous to the concept of temperature in thermodynamics. Therefore, $v_{\text {ave }}$ can express the activity of a swarm and provide useful information for the construction of adaptive PSO.

\subsection{Relationship between success or failure of} search and average velocity of particles

As described above, a particle transfers by means of the transfer vector $v_{i j}$ generated by Equation (1), thus $\left|v_{i j}\right|$ is the step size of the transfer. If the parameter values are not appropriate, then the absolute value of 


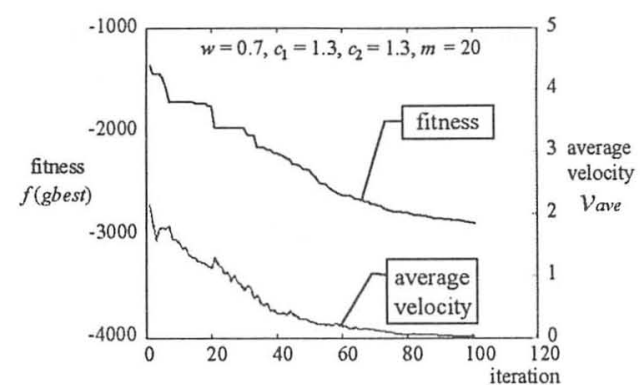

(a) Appropriate parameter setting

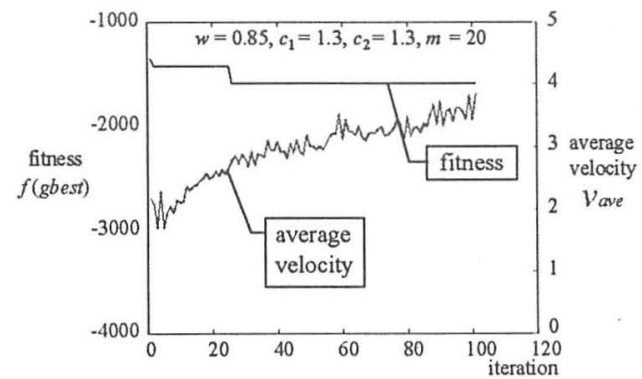

(b) Divergent parameter setting

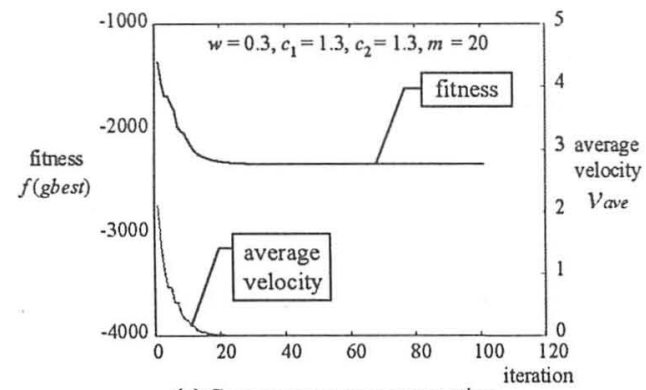

(c) Convergent parameter setting

Fig. 3: Relationship between success or failure of search and average velocity of particles

velocity $\left|v_{i j}\right|$ can increase or decrease rapidly. Neither of these cases is desirable from the viewpoint of the search process. These cases are included in the examples plotted in Figure 3. The figure shows the results obtained by applying PSO with constant parameters to the $2^{n}$ minima problem ( 50 dimensions) for a swarm of population 20. The fitness of gbest $f$ (gbest) and the average of the absolute value of velocity $v_{\text {ave }}$ are plotted.

For the case shown in Figure 3(a), where the average velocity of particles gradually decreases, a good solution tends to be obtained. On the other hand, for the case shown in Figure 3(b), for which the parameters have a divergent character, the average velocity of particles gradually increases, and the search ends in failure. For the case shown in Figure 3(c), the parameters are overly convergent, and the particles become almost static following iteration 20 . As indicated by the typical examples shown here, a qualitative relationship exists between the average velocity of particles and the success or failure of the search.

Although only one example of the $2^{n}$ minima problem was shown in this paper, we verified by other bench-

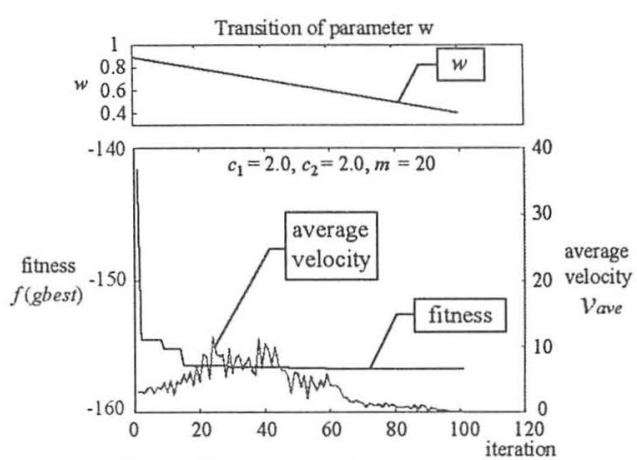

(a) Low-dimensional problem ( 2 dimensions) Transition of parameter $\mathrm{w}$

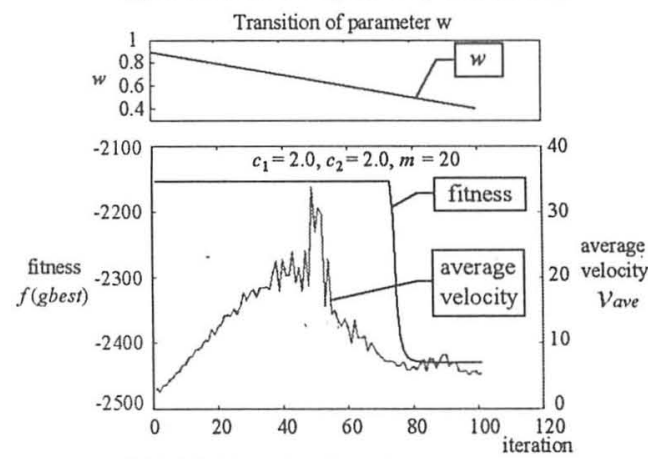

(b) High-dimensional problem (100 dimensions)

Fig. 4: Simulation results of the LDIWA

mark problems that such a relationship holds. Rather than being peculiar to the $2^{n}$ minima problem, this qualitative relationship is thought to be applicable to numerous problems.

\subsection{Simulation results for LDIWA}

Next, we examine the dependency of the decrease in the search ability of the LDIWA for large-scale problems upon the average velocity of particles. Figure 4 shows the results obtained by applying the LDIWA to the $2^{n}$ minima problems of 2 dimensions and 100 dimensions, respectively, where the population of the swarm is 20 , and the fitness of gbest and the average of the absolute value of velocity of particles are plotted as in Figure 3.

While the average velocity of particles for the smallscale problem is at most 10 , that for the large-scale problem reaches over 30 at its peak values. For the test problem (3), the space to be searched is $-5 \leq x_{i} \leq$ 5. Therefore, when the average velocity of particles is over 10, particles often leave the search space. Figure 4 clarifies that for the same parameter values, the average velocity of particles in a large-scale problem is larger than that in a small-scale problem.

Therefore, the LDIWA is concluded to be less effective for large-scale problems, compared to small-scale problems, because when the LDIWA is applied to a large-scale problem the search finishes before the search phase shifts from diversification to intensification. 


\section{Activity-Feedback Feedback Adaptive PSO Algorithm}

It has been shown in this study that the success or failure of a search is related to the average of the absolute value of velocity of particles. Therefore, in this section, a new algorithm is proposed in which parameters are tuned so as to control the average velocity of particles.

Although the search ability of LDIWA decreases when applied to a large-scale problem, the strategy of LDIWA, i.e., gradually shifting the search phase from diversification to intensification as the iteration progresses, appears to be effective for optimization. Therefore, we propose a new algorithm in which parameters are tuned adaptively in order to maintain the ideal velocity, as shown in Figure 5.

In this algorithm, if the current average velocity of particles is larger than the goal velocity, parameters are shifted to convergent values. Otherwise, parameters are sifted to divergent values (see Figure 6). By applying such a strategy, the average velocity of particles will follow the goal velocity. In Figure 5, the proposed ideal velocity reaches 0 before the end of the search, because the search is intended to be intensive in the final stage.

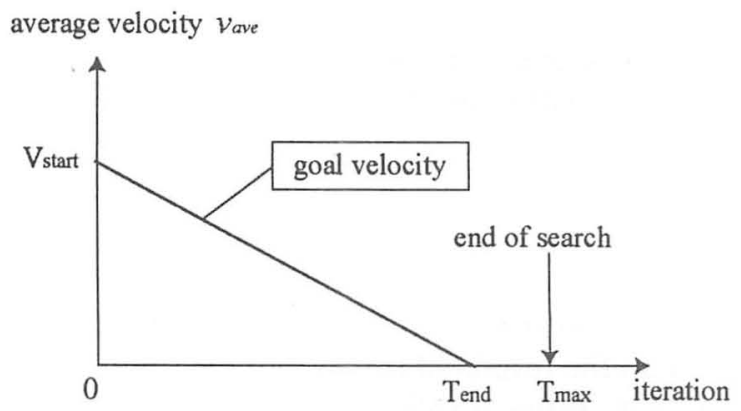

Fig. 5: Goal velocity for the proposed adaptive PSO

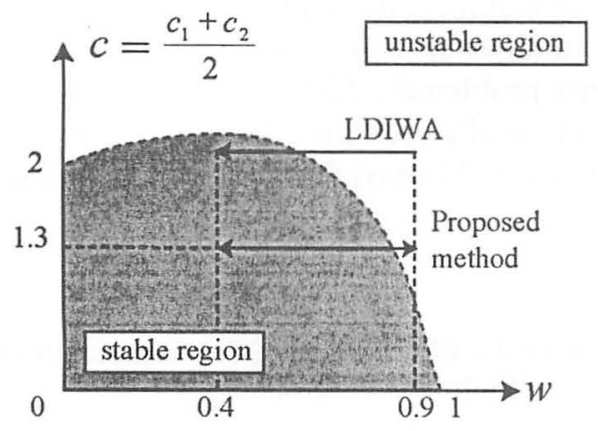

Fig. 6: Parameter tuning in the proposed algorithm and in the LDIWA

\section{[Activity-Feedback Feedback Adaptive PSO} Algorithm]

Step 0: Preparation : Decide the parameters $w^{0}, \Delta w$, $w_{\max }, w_{\min }, c_{1}, c_{2}, m, \mathrm{~T}_{\max }, \mathrm{T}_{\text {end }}, \mathrm{V}_{\text {start }}$ and $V_{\text {init }}$.

Step 1: Initialization : Generate the initial position of each particle $x_{i}^{0}$ randomly, and set the initial velocity of each particle $v_{i j}^{0}$ in the range of $\left[-\mathrm{V}_{\text {init }}, \mathrm{V}_{\text {init }}\right]$ randomly. Set goal velocity ${ }^{1}=$ $\mathrm{V}_{\text {start }}$ and $k=0$, where $k$ is an iteration counter.

Step 2: Evaluation : Calculate the evaluated value $f\left(x^{k}\right)$ for each solution $x^{k}$.

Step 3: Updating of pbest and gbest : Compare each evaluated value $f\left(x^{k}\right)$ with the current pbest. If $f\left(x^{k}\right)<f\left(\right.$ pbest $\left.^{k-1}\right)$, set pbest ${ }^{k}=x^{k}$. Otherwise, set pbest ${ }^{k}=$ pbest $^{k-1}$. If $k=0$, set pbest $t^{0}=x^{0}$ without comparison. Compare and update gbest, in the same way as pbest.

Step 4: Transfer of each particle : According to Equations (1) and (2), each particle $x^{k}$ is transferred to $x^{k+1}$. Furthermore, $v_{\text {ave }}{ }^{k+1}$ is calculated by Equation (4).

Step 5: Modification of parameters : Compare the current average velocity of particles $v_{\text {ave }}^{k+1}$ with goal velocity ${ }^{k+1}$. If $v_{\text {ave }}{ }^{k+1}>$ goal velocity ${ }^{k+1}$, then $w^{k+1}=\max \left\{w^{k}-\Delta w, w_{\min }\right\}$. Otherwise $w^{k+1}=\min \left\{w^{k}+\Delta w, w_{\max }\right\}$.

Step 6: Modification of goal velocity : Modify goal velocity according to the following equation: goal velocity ${ }^{k+2}=$ goal velocity $^{k+1}-$ $\mathrm{V}_{\text {start }} / \mathrm{T}_{\text {end }}$

If counter $k$ does not reach the predetermined maximum number of iterations $\mathrm{T}_{\max }$, set $k=k+1$ and return to Step 2.

Table 2: Parameters of LDIWA and proposed algorithm for simulation

\begin{tabular}{c|c||c}
\hline \hline \multirow{4}{*}{ LDIWA } & $w_{\max }$ & 0.9 \\
\cline { 2 - 3 } & $w_{\min }$ & 0.4 \\
\cline { 2 - 3 } & $c_{1}$ & 2.0 \\
\cline { 2 - 3 } & $c_{2}$ & 2.0 \\
\cline { 2 - 3 } & $m$ & 20 \\
\cline { 2 - 3 } & $T_{\max }$ & 1000 \\
\hline \hline \multirow{4}{*}{$\begin{array}{l}\text { Proposed } \\
\text { algorithm }\end{array}$} & $V_{\text {init }}$ & 1 \\
\cline { 2 - 3 } & $w^{0}$ & 0.9 \\
\cline { 2 - 3 } & $\boldsymbol{D w}_{\mathrm{w}}$ & 0.1 \\
\cline { 2 - 3 } & $w_{\max }$ & 0.9 \\
\cline { 2 - 3 } & $w_{\min }$ & 0.4 \\
\cline { 2 - 3 } & $c_{1}$ & 1.3 \\
\cline { 2 - 3 } & $c_{2}$ & 1.3 \\
\cline { 2 - 3 } & $T_{\max }$ & 20 \\
\cline { 2 - 3 } & $T_{\text {end }}$ & 1000 \\
\cline { 2 - 3 } & $V_{\text {start }}$ & 10 \\
\cline { 2 - 3 } & $V_{\text {init }}$ & 20 \\
\hline \hline
\end{tabular}


Table 3: Application results for $2^{n}$ minima ( $\mathrm{T}_{\max }=1000$, Size of swarm $m=20$ )

\begin{tabular}{c|c||c|c||c|c||c|c||c|c}
\hline \hline \multicolumn{3}{c||}{} & \multicolumn{2}{c||}{ Mean value } & \multicolumn{2}{c||}{ Best value } & \multicolumn{2}{c|}{ Worst value } & \multicolumn{2}{c}{ Standard deviation } \\
\hline $\begin{array}{c}\text { Dim. } \\
\text { (n) }\end{array}$ & $\begin{array}{c}\text { Optimal } \\
\text { value }\end{array}$ & LDIWA & $\begin{array}{c}\text { Proposed } \\
\text { algorithm }\end{array}$ & LDIWA & $\begin{array}{c}\text { Proposed } \\
\text { algorithm }\end{array}$ & LDIWA & $\begin{array}{c}\text { Proposed } \\
\text { algorithm }\end{array}$ & LDIWA & $\begin{array}{c}\text { Proposed } \\
\text { algorithm }\end{array}$ \\
\hline \hline 10 & -783 & -758 & -772 & -783 & -783 & -642 & -727 & 25 & 16 \\
20 & -1567 & -1410 & -1468 & -1538 & -1567 & -1256 & -1340 & 62 & 51 \\
30 & -2350 & -1902 & -2105 & -2250 & -2264 & -1173 & -1925 & 167 & 68 \\
40 & -3133 & -1650 & -2724 & -2370 & -2905 & -780 & -2395 & 399 & 91 \\
50 & -3917 & -1506 & -3334 & -2399 & -3549 & -869 & -3081 & 313 & 97 \\
60 & -4700 & -1610 & -3915 & -2666 & -4214 & -1171 & -3618 & 280 & 118 \\
70 & -5483 & -1740 & -4470 & -2589 & -4831 & -1125 & -4048 & 303 & 148 \\
80 & -6267 & -1850 & -5011 & -2518 & -5410 & -1183 & -4621 & 276 & 144 \\
90 & -7050 & -2007 & -5507 & -2968 & -5904 & -1254 & -5099 & 287 & 169 \\
100 & -7833 & -2101 & -5937 & -2964 & -6480 & -1276 & -5262 & 366 & 211 \\
\hline \hline
\end{tabular}

\section{Simulation Results for Benchmark Problems}

The LDIWA and the proposed adaptive PSO are applied to typical benchmark problems $\left(2^{n}\right.$ minima problem) in order to verify the search ability of the proposed adaptive PSO. The formulations and the shapes of the benchmark problems are shown in Appendix.

While the proposed algorithm does not require that parameters $w, c_{1}$ and $c_{2}$ be determined before the search begins, $V_{\text {start }}$ and $T_{\text {end }}$ in Figure 5 must be determined. For the benchmark problems, we selected $\mathrm{V}_{\text {start }}=x_{i \max }-x_{i \min }$, because the search spaces for the benchmark problems are given by $x_{i \min } \leq x_{i} \leq$ $x_{i} \max$. Since there is no clear policy for deciding $\mathrm{T}_{\text {end }}$, we set $\mathrm{T}_{\text {end }}$ as $80 \%$ of the entire number of iterations $\mathrm{T}_{\max }$ in this simulation. The details of the values of the other parameters for both the LDIWA and the proposed adaptive PSO is listed in Table 2.

Table 3 lists the comparison of the results of applying the LDIWA and the proposed algorithm to the $2^{n}$ minima problem as formulated in Equation (3). In comparison with the LDIWA, the proposed adaptive PSO has higher optimality especially for large-scale problems.

Figure 7 shows an example of the results of applying the proposed algorithm to the $2^{n}$ minima problem (the dimension of the problem $n=100$, the size of the swarm $\left.m=20, \mathrm{~T}_{\max }=200\right)$. The transitions of parameter $w$, the best fitness $f\left(g b e s t\right.$ ), and the average velocity $v_{\text {ave }}$ are shown in this figure. This figure shows that adaptive tuning of parameter $w$ enables the average velocity of particles to follow the goal velocity.

Based on the simulation results, the proposed algorithm is also confirmed to have higher optimality than the LDIWA.

\section{Conclusions}

In this paper, we analyzed the functioning of the Particle Swarm Optimization (PSO) algorithm by viewing the swarm as a discrete dynamic system and then considering the stability thereof. In addition, we consid-

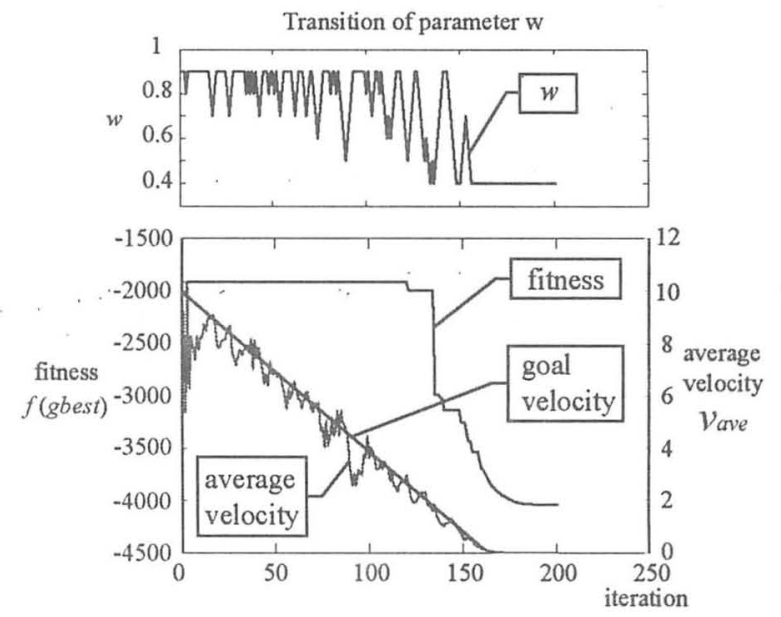

Fig. 7: Transitions of parameter $w, v_{\text {ave }}, f(g b e s t)$ in the proposed algorithm for $2^{n}$ minima problem

ered the qualitative and quantitative relationships between the behavior of the algorithm and the success or failure of the search.

Based on the results of these analyses, while the average absolute value of velocity of all of the particles was defined as an index to understand the activity of all of the particles, an adaptive PSO algorithm that feeds back the information of the average velocity of particles was proposed.

The search ability of the proposed algorithm was verified by simulation using typical benchmark problems ( $2^{n}$ minima problems). Although only four examples of application results are shown in the present paper, similar results were obtained for other benchmark problems.

\section{References}

[1] Kennedy J., Eberhart R. C.: "Swarm Interlligence", Morgan Kaufmann Publishers (2001)

[2] Yasuda K., Ide A. and Iwasaki N.: "Stability Analysis of Particle Swarm Optimization", Proceedings of The Fifth Metaheuristics International Conference, MIC0334 (2003) 